\title{
Caractérisation des modes d'instabilités en aval d'une cavité ouverte de forme variable
}

\author{
Mourad Bouterra $^{1, a}$, Afif El Cafsi ${ }^{1}$, Ali Belghith ${ }^{1}$, François Lusseyran ${ }^{2}$ \\ Et Pierre Gougat ${ }^{2}$ \\ 1 Département de physique, Facultés des Sciences de Tunis, Campus Universitaire, 1060 Tunis, Tunisie \\ 2 LIMSI-CNRS Bât. 508, BP 133, 91403 Orsay Cedex, France
}

Reçu le 9 juin 2008, accepté le 14 octobre 2009

\begin{abstract}
Résumé - Dans ce travail, on s'intéresse à l'étude et à la caractérisation expérimentale par la technique vélocimétrie laser à effet Doppler, du phénomène de détachement tourbillonnaire lors de l'interaction couche limite cavité. L'étude est effectuée en changeant la longueur et la hauteur de la cavité, ainsi que la vitesse de l'écoulement, afin de modifier la nature des structures présentes à l'intérieur de la cavité. La variation du nombre de Strouhal en fonction du nombre de Reynolds conduit à un regroupement pour chaque mode d'instabilité, permettant de bien séparer les modes les uns des autres.
\end{abstract}

Mots clés : Cavité / échappement tourbillonnaire / mode fréquentiel / analyse spectrale / nombre de Strouhal

\begin{abstract}
Characterization of instabilities modes downstream an open cavity of various shape. In this paper, we have studied and characterized experimentally by the technique of measure Laser Doppler Velocimetry (LDV) the vortex shedding frequency. The study is conducted by changing the cavity length and height and the external flow velocity and therefore the flow patterns inside the cavity. The variation of the Strouhal number according to the Reynolds number, led us to a regrouping for every mode, permitting to really separate the mode the some of the other.
\end{abstract}

Key words: Cavity / vortex structures / frequency mode / spectral analysis / Strouhal number

\section{Introduction}

L'écoulement en cavité est un paradigme abondamment étudié pour les écoulements autour de discontinuités structurelles d'engins [1]. Ce type d'écoulement est principalement gouverné par le développement d'une couche de cisaillement [2]. Celle-ci est le siège d'instabilités, donnant naissance à des structures tourbillonnaires organisées. L'identification de ces structures représente un élément important de la dynamique des écoulements réels.

La majorité des études antérieures rapportent que l'écoulement de cavité ne modifie pas fondamentalement le comportement naturel de la couche de mélange. Les oscillations, initialement présentes, à l'intérieur de la cavité, sous forme de modes hydrodynamiques de type Kelvin Helmholtz, subissent une forte amplification

\footnotetext{
a Auteur pour correspondance :

mourad.bouterra@fst.rnu.tn
}

lorsqu'elles sont soumises à la présence du bord aval de la cavité. Toutefois, ce mécanisme auto-entretenu n'évolue pas nécessairement vers la formation de structures tourbillonnaires telles que celles créées dans la couche de mélange en développement libre. Dans la revue bibliographique de Rockwell et Naudascher [2] consacrée à la formation des écoulements de cavité, ce mode d'oscillation est identifié comme mode de cisaillement.

L'étude de Sarohia [3], sur des cavités axisymétriques, où la couche limite incidente est laminaire, montre que la présence du bord aval conduit à une forte amplification des instabilités de la couche de mélange. Cette étude montre la présence de deux modes d'instabilités dont la fréquence correspond à des valeurs du nombre de Strouhal modulées par la longueur de la cavité, l'épaisseur de la couche de mélange incidente $(\delta)$ et dans une moindre mesure, par la profondeur de la cavité et le nombre de Reynolds $\left(\operatorname{Re}_{\delta}\right)$. Sur la base de ces différentes observations, Sarohia [3] a cherché à déterminer les domaines d'apparition des instabilités. Il conclut que les 


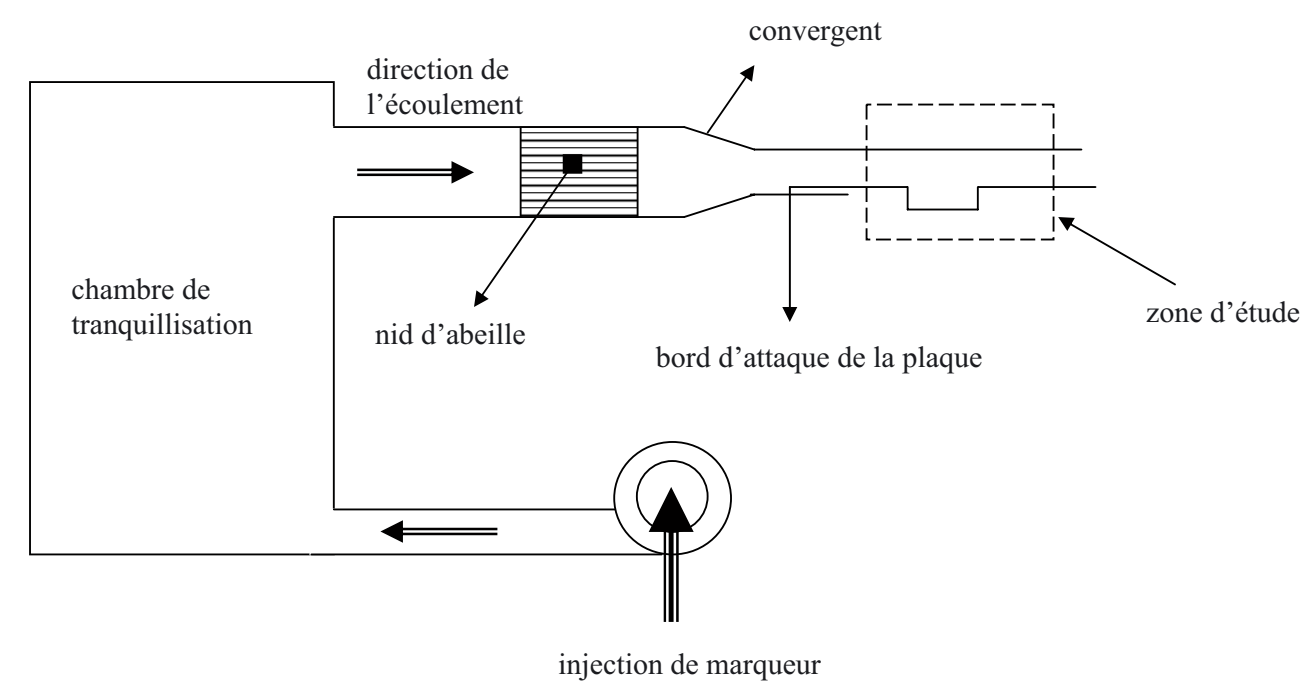

Fig. 1. Dispositif expérimental.

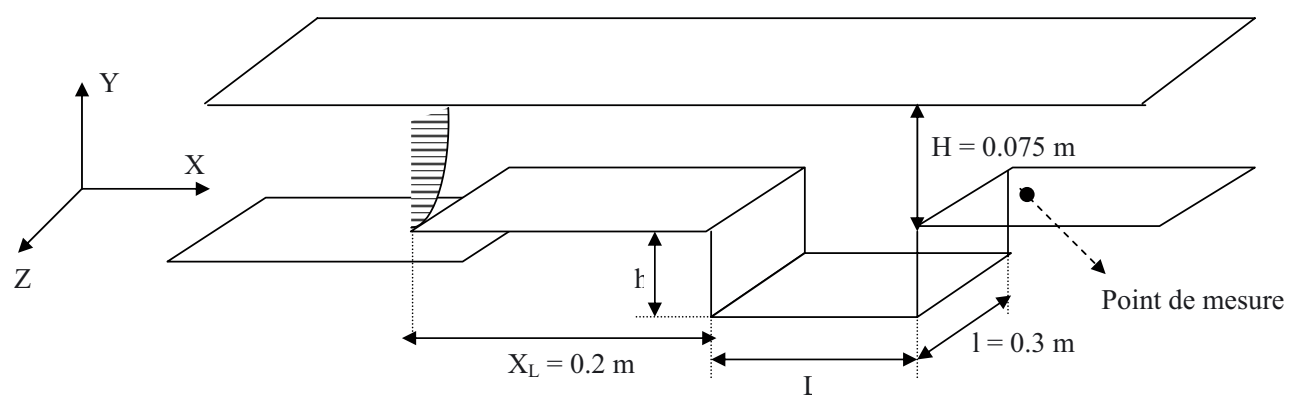

Fig. 2. Schéma de la veine d'essai.

caractéristiques $\delta$ et $R e_{\delta}$ de la couche limite incidente imposent une longueur et une profondeur minimales pour l'établissement des instabilités. Sarohia [3] ajoute que les fortes modifications dues à la présence du bord aval ont un effet stabilisant qui tend à retarder la formation de tourbillons cohérents et la transition de la couche de mélange vers la turbulence. Les observations effectuées en écoulement turbulent par Ethembabaoglu [4] montrent une distribution des instabilités selon trois modes, correspondant globalement à des valeurs constantes du nombre de Strouhal et ne présentant pas de relation harmonique. L'auteur montre également que la diminution de l'allongement longitudinal conduit à une réduction de la fréquence des instabilités moins marquée que celle observée en régime laminaire par Sarohia [3]. Il apparaît également que les différents modes peuvent coexister et que leurs niveaux relatifs évoluent en fonction des paramètres de la configuration.

La couche de cisaillement a été identifiée, dans la plupart des publications, comme le processus de formation des structures tourbillonnaires. Bien que l'action de ces dernières sur la dynamique de l'écoulement en cavité soit déterminante, il existe peu de données quantitatives les caractérisant. Dans le présent travail, on se propose, d'une part, de présenter la dynamique d'un écoulement (à faible vitesse) résultant de l'interaction entre une couche limite laminaire et une cavité de rapport d'aspect variable, d'autre part, de quantifier l'étude par une analyse de l'évolution en fonction du rapport d'aspect, des modes fréquentiels mesurés par LDV. L'accent sera mis sur la variation du nombre de Strouhal, en fonction du nombre de Reynolds.

\section{Dispositif expérimental}

Dans nos expériences, l'écoulement, supposé incompressible, s'effectue à basse vitesse. La couche de cisaillement qui se forme à l'affleurement amont de la cavité est théoriquement instable dès l'établissement de l'écoulement [5].

Le schéma de principe de l'installation expérimentale utilisée, pour l'étude de l'interaction couche limite cavité, est représenté sur la figure 1. L'écoulement est créé par un ventilateur centrifuge placé en amont de la chambre de tranquillisation. L'injection de marqueur s'effectue à l'entrée du ventilateur. Un conduit axial se terminant par un nid d'abeilles et un convergent amènent l'écoulement vers la section d'essai. Cet écoulement à caractère uniforme, arrive parallèlement à une plaque plane mince, dont le bord d'attaque, de forme elliptique, est orthogonal à la vitesse. Une longueur de la plaque, de $200 \mathrm{~mm}$ permet de fournir une couche limite laminaire. La veine d'essai de ce dispositif (Fig. 2) a une section rectangulaire, de 


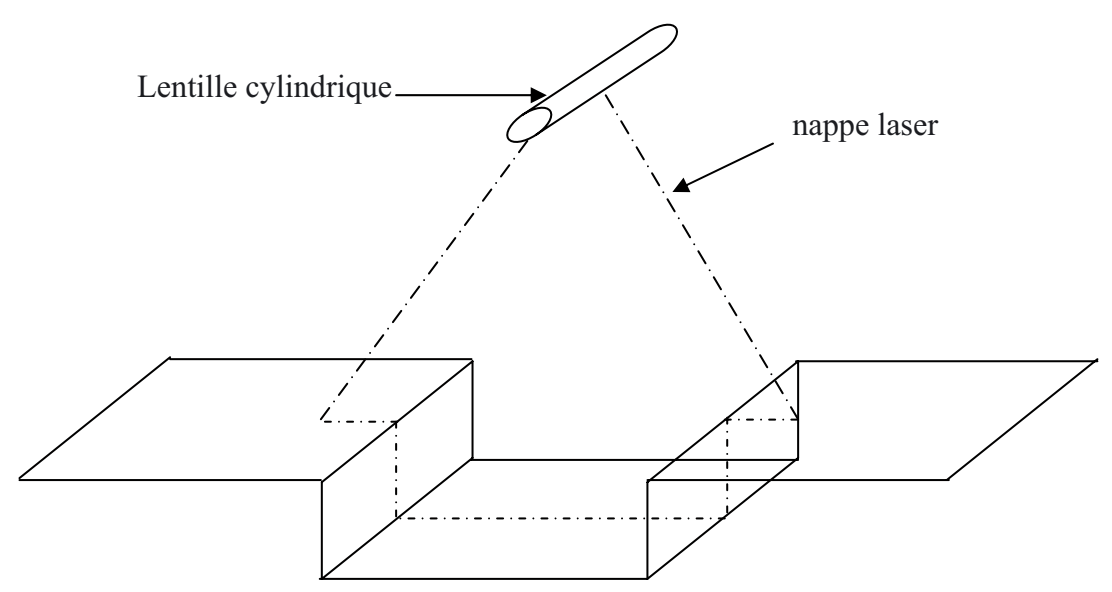

Fig. 3. Exemple d'éclairement de la cavité par une nappe laser.

Tableau 1. Dimension caractéristique des cavités ayant $L$ constante.

\begin{tabular}{cccc}
\hline & $R=0,5$ & $R=1$ & $R=2$ \\
\hline$h(\mathrm{~mm})$ & 200 & 100 & 50 \\
$L(\mathrm{~mm})$ & 100 & 100 & 100 \\
\hline
\end{tabular}

Tableau 2. Dimension caractéristique des cavités ayant $h$ constante.

\begin{tabular}{cccc}
\hline & $R=1$ & $R=1,5$ & $R=2$ \\
\hline$h(\mathrm{~mm})$ & 50 & 50 & 50 \\
$L(\mathrm{~mm})$ & 50 & 75 & 100 \\
\hline
\end{tabular}

$75 \mathrm{~mm}$ de hauteur sur $300 \mathrm{~mm}$ de largeur, et une longueur totale de l'ordre de $600 \mathrm{~mm}$. Cette veine est fabriquée en plexiglas, pour faciliter les prises de vue de l'extérieur et l'éclairement de l'écoulement. À la sortie de la soufflerie, l'air est rejeté dans la salle de mesure. Le ventilateur utilisé dans ce système permet d'avoir des écoulements caractérisés par des vitesses, pouvant atteindre $5 \mathrm{~m} . \mathrm{s}^{-1}$. La hauteur $h$ de la cavité varie entre $50 \mathrm{~mm}$ et $200 \mathrm{~mm}$. Son envergure $l=300 \mathrm{~mm}$, est fixée par les parois latérales de la soufflerie. En plus des nombres de Reynolds et de Strouhal, on définit deux autres nombres sans dimensions, à savoir le rapport d'envergure $F=l / h$ compris entre 1,5 et 6 , et le rapport d'aspect de la cavité $R=L / h$ compris entre 0,5 et 2 . Les différentes valeurs du rapport d'aspect ont été obtenues en faisant varier, soit la hauteur $h$ de la cavité, soit sa longueur $L$. Les dimensions des différentes cavités étudiées, sont données dans les tableaux 1 et 2 .

L'origine du système de coordonnées est prise sur le bord d'attaque à mi-envergure, l'axe $x$ est dans la direction de l'écoulement, l'axe $y$ normal à la plaque amont et l'axe $z$ selon l'envergure de la cavité. On a vérifié que la paroi supérieure de la section d'essai n'a pas d'influence sur le développement de la couche de cisaillement ni sur l'écoulement. L'épaisseur de la couche limite qui se développe sur cette paroi est inférieure à $10 \mathrm{~mm}$ et n'a pas d'influence sur l'écoulement extérieur le long des deux directions longitudinale et transversale. L'épaisseur a essentiellement pour effet d'accélérer le fluide au-dessus de la cavité par conservation du débit. La vitesse amont $U_{\infty}$ est mesurée à $102 \mathrm{~mm}$ en amont de la cavité et 25,5 mm au-dessus de la plaque amont. Dans cette étude, l'apparition et la propagation de tourbillons à grande échelle semblent être relativement peu affectées par les effets de confinement.

\section{Résultats}

\subsection{Mise en évidence du phénomène d'échappement tourbillonnaire}

La visualisation est une étape assez importante dans l'étude des écoulements fluides. C'est une phase préliminaire nécessaire pour la caractérisation qualitative de la structuration de l'écoulement. La visualisation permet de donner, en première approche, une idée globale sur l'écoulement, de focaliser les zones à fortes activités tourbillonnaires et également de délimiter les zones à explorer par les techniques locales de mesure de vitesses. Pour ce travail, nous avons réalisé des visualisations de l'écoulement aussi bien par de la fumée de spectacle que par de la fumée d'encens [6-8], la cavité étant éclairée par une nappe laser conformément au schéma de la figure 3. Les prises de vue sont enregistrées (300 paires d'images) par une caméra CCD Pulnix9701 (30 images par seconde), de résolution $768 \times 484$ pixels sur 8 bits, reliée directement à un ordinateur par une carte d'interface spécifique.

Sur la figure 4, on présente la visualisation d'un instantané de l'écoulement, dans la cavité large, réalisée avec de la fumée de spectacle pour une vitesse $U_{\infty}$ de $1,75 \mathrm{~m} . \mathrm{s}^{-1}$. Cette fumée est obtenue par injection d'huile sur la plaque chaude placée en amont de la cavité. Cette technique a l'avantage de donner une fumée dense et homogène. La bonne densité des particules à l'intérieur de la cavité, permet de garder une certaine continuité des niveaux de gris de l'image obtenue $[8,9]$.

Sur cette visualisation apparaissent les différentes structures de l'écoulement. L'écoulement principal est 


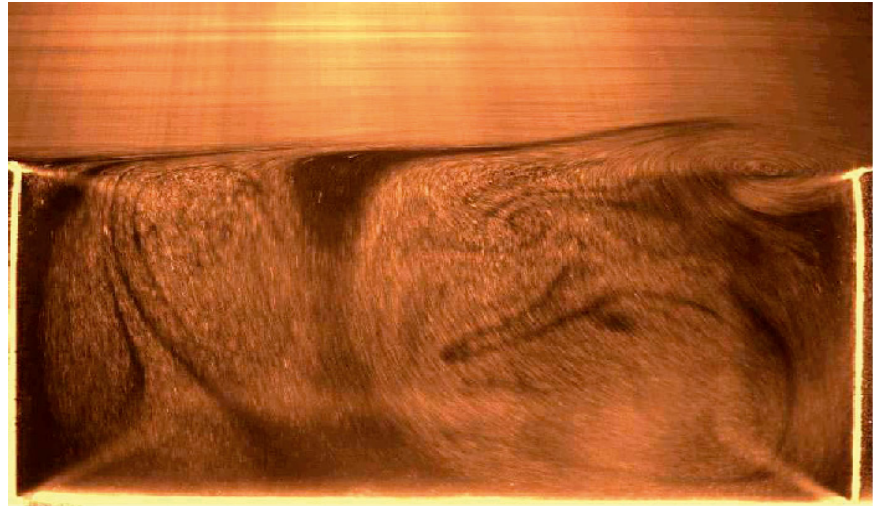

Fig. 4. Visualisation de l'écoulement dans la cavité par la fumée du spectacle.

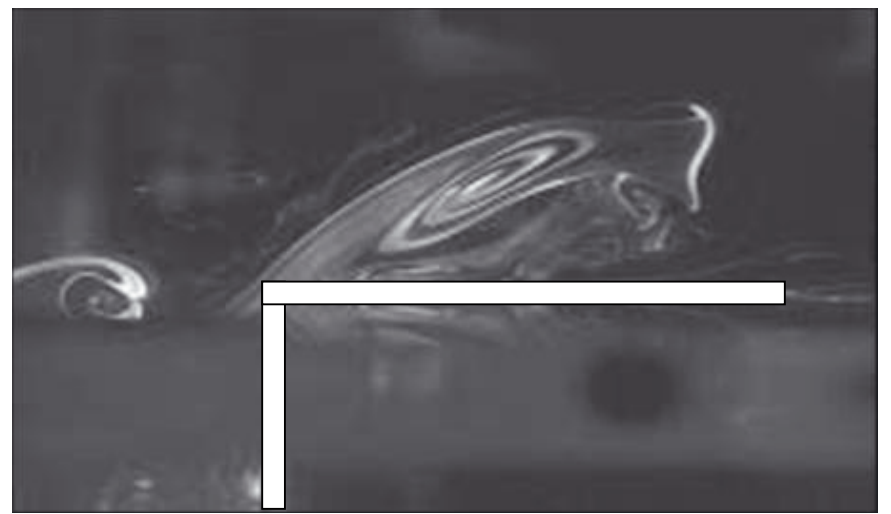

Fig. 5. Visualisation de l'échappement tourbillonnaire de la cavité par la fumée d'encens.

séparé des vortex de la cavité par une couche de cisaillement. Cette zone, siège de forts gradients de vitesse, est marquée par les instabilités de cisaillement, se développant jusqu'à former des rouleaux de fumée, qui viennent régulièrement se briser sur le coin arrière de la cavité. Une part de la vorticité de ces tourbillons est entraînée par le tourbillon principal de la cavité. Ce tourbillon principal, situé près de la paroi face au vent, tourne dans le sens des aiguilles d'une montre, alors que des mouvements tourbillonnaires, de sens contraire et de plus faible intensité, se produisent dans le reste de la cavité. Dans un premier temps, on constate que le tourbillon principal se déplace à l'intérieur de la cavité sous les effets de frottements visqueux. Cette structure va entraîner le reste du fluide en mouvement et sa taille va augmenter. Dans ce cas, son diamètre est de l'ordre de la hauteur de la cavité. Cette évolution montre que le fluide pénètre d'abord dans la cavité au niveau de la paroi face au vent, puis il remonte vers le milieu pour pénétrer de nouveau dans la cavité. Ces tourbillons seront évacués par la suite vers l'aval de la cavité $[7,9]$. Nous avons réalisé également des visualisations à l'aval de la cavité. La figure 5 a été obtenue avec de la fumée se dégageant d'un bâton d'encens placé dans la cavité, pour une vitesse $U_{\infty}$

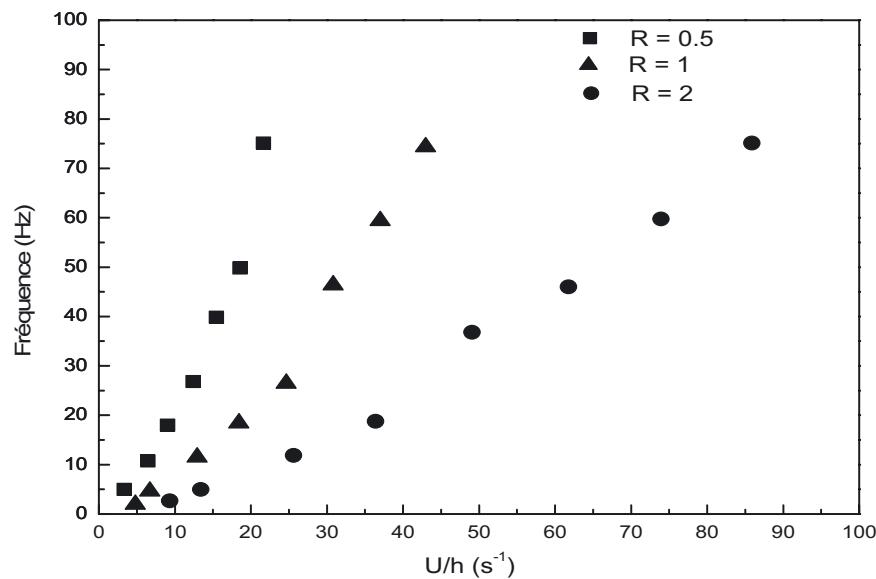

Fig. 6. Variation des fréquences d'échappement tourbillonnaire en fonction du rapport $U / h$ pour des cavités ayant la même longueur $L$.

de $1,27 \mathrm{~m} \cdot \mathrm{s}^{-1}$. Cette figure met en évidence des tourbillons dans cette région, ainsi que leurs entraînements par l'écoulement principal. L'analyse des images obtenues, en tenant compte du facteur de grossissement, nous a permis de déduire une vitesse de translation sensiblement égale à $U_{\infty}$. Pour mieux caractériser la dynamique spatio-temporelle des mouvements tourbillonnaires, nous avons réalisé des mesures de vitesse par vélocimétrie laser Doppler LDV en certains points de la cavité et de la zone d'échappement.

\subsection{Variation des fréquences en fonction de la vitesse débitante}

Dans la zone aval de la cavité, nous avons effectué des mesures par LDV [10] de la composante longitudinale de la vitesse. Ces mesures ont été faites pour une large gamme de valeurs de la vitesse débitante $U_{\infty}$, allant de 0 jusqu'à $5 \mathrm{~m} \cdot \mathrm{s}^{-1}$. Le point de mesure situé dans la zone d'échappement, se trouve à $15 \mathrm{~mm}$ du bord de fuite et à $15 \mathrm{~mm}$ également de la paroi, comme le montre la figure 2. Le point de mesure se situe dans l'écoulement résultant de la couche de cisaillement pour subir toute perturbation due, d'une part, à la couche de mélange essentiellement localisée au-dessus de la cavité, d'autre part, aux structures tourbillonnaires intra-cavitaires. Nous avons utilisé comme traceurs, les particules du dioctylphtalate, injectées au niveau du ventilateur de la soufflerie. Les données obtenues par enregistrement, ont été traitées par le logiciel Dataview développé par la société Aerometrics. Les mesures de vitesse par LDV dans la zone d'échappement tourbillonnaire, ont été réalisées pour plusieurs cavités caractérisées par différentes valeurs du rapport d'aspect $R$. La figure 6 est relative aux résultats obtenus pour les cavités ayant une même longueur $L$. Nous remarquons que pour le cas d'un rapport d'aspect égal à 0,5 , l'échappement tourbillonnaire ne se produit plus à partir d'une vitesse supérieure à $4 \mathrm{~m} \cdot \mathrm{s}^{-1}$. 


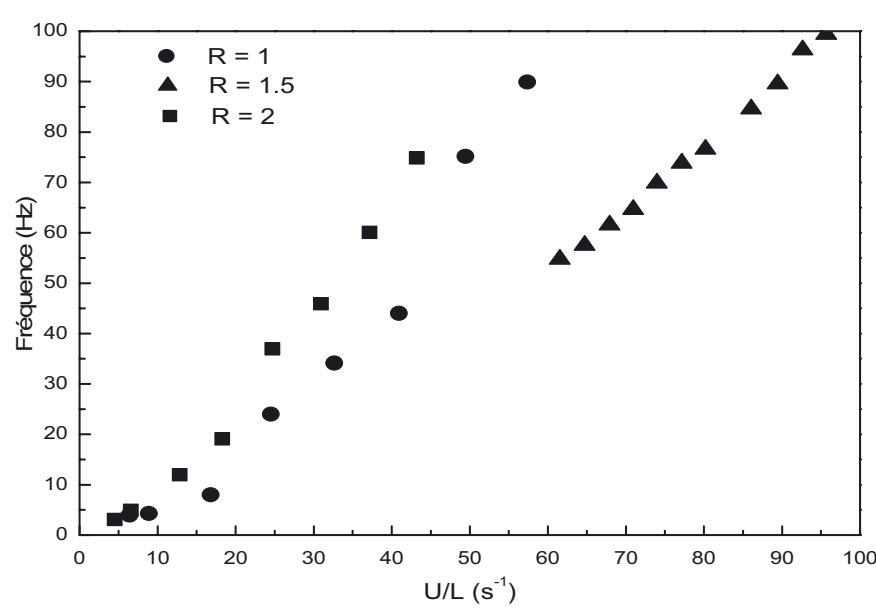

Fig. 7. Variation des fréquences d'échappement tourbillonnaire en fonction du rapport $U / L$ pour des cavités ayant la même hauteur $h$.

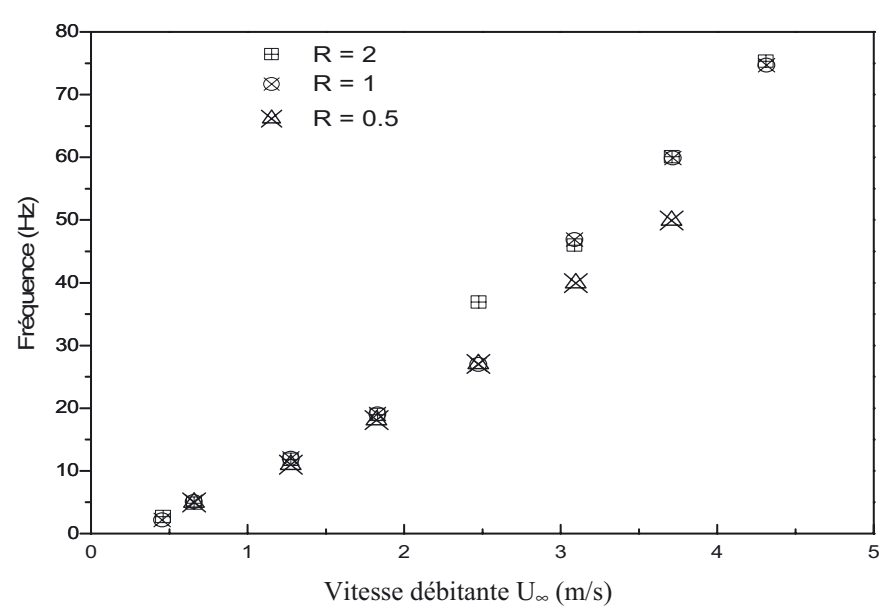

Fig. 8. Variations des fréquences en fonction des vitesses $U_{\infty}$ pour les trois cavités.

Sur la figure 7, nous présentons les fréquences d'échappement mesurées, en fonction du rapport $\frac{U}{L}$ pour les cavités ayant une même hauteur $h$. Pour le cas du rapport d'aspect égal à 1 , nous constatons qu'il n'y a pas d'échappement tourbillonnaire pour des vitesses inférieures à $3 \mathrm{~m} . \mathrm{s}^{-1}$. La comparaison des valeurs des fréquences d'échappement, obtenues pour une même vitesse débitante et pour différentes valeurs du rapport d'aspect, montre que la variation de la hauteur de la cavité a une influence assez limitée. Par contre, l'analyse des résultats obtenus, pour le cas des cavités ayant une même hauteur, permet de conclure que la longueur $L$ de la cavité est la dimension principale, qui contrôle l'échappement tourbillonnaire. Ceci est confirmé par des résultats antérieurs $[11,12]$.

Dans le but de nous assurer de ce résultat, nous avons tracé les courbes de variations des fréquences dominantes en fonction des vitesses débitantes, pour les trois cavités ayant en commun une même longueur $L$ égale à $100 \mathrm{~mm}$. Pour chacune de ces courbes (Fig. 8), nous observons une croissance plutôt linéaire de la fréquence par rapport à la
Tableau 3. Paramètres de l'ajustement linéaire pour les trois cavités.

\begin{tabular}{lcccc}
\hline & $\alpha$ & $f_{0}$ & $U_{C}=-\frac{f_{0}}{\alpha}\left(\mathrm{m} . \mathrm{s}^{-1}\right)$ & $R e_{C}=\frac{U_{C} L}{\nu}$ \\
\hline$R=0,5$ & 15,10 & $-7,56$ & 0,50 & 3337 \\
$R=1$ & 18,80 & $-10,87$ & 0,57 & 3853 \\
$R=2$ & 18,79 & $-9,60$ & 0,51 & 3406 \\
\hline
\end{tabular}

vitesse débitante. Le résultat de l'ajustement linéaire de ces courbes par des fonctions de la forme $f=\alpha U_{\infty}+f_{0}$, déterminé par le critère des moindres carrés, est donné dans le tableau 3. Le choix de l'ajustement est justifié, d'une part, par l'allure des courbes obtenues et d'autre part, par des résultats antérieurs [11]. Nous constatons, pour les trois cas étudiés, que les valeurs des pentes $\alpha$ sont positives et que les valeurs des ordonnées à l'origine $f_{0}$ sont négatives.

Nous constatons, également, que le rapport $-\frac{f_{0}}{\alpha}$ donne toujours une valeur de l'ordre de 0,5 . Ce rapport homogène à une vitesse, nous permet de définir une vitesse critique $U_{C}$ relative à l'apparition du phénomène de détachement tourbillonnaire. Pour une longueur de cavité constante, cette valeur critique observée n'est pas affectée par la variation de la hauteur de la cavité. Ce résultat confirme le rôle joué par la variation de la longueur de la cavité comme dimension principale dans le contrôle du détachement tourbillonnaire. Nous retenons alors, conformément aux études antérieures, la longueur $L$ de la cavité comme dimension caractéristique pour le calcul des nombres sans dimensions.

\subsection{Relation entre la couche de cisaillement et les modes d'instabilités}

Le rapport d'aspect $R=L / h$ de la cavité est maintenu constant et égal à $2(L=100 \mathrm{~mm}$ et $h=50 \mathrm{~mm})$, la vitesse débitante $U_{\infty}$, varie de $0,1 \mathrm{~m} . \mathrm{s}^{-1}$ à $2,6 \mathrm{~m} . \mathrm{s}^{-1}$. À partir d'enregistrements de $150 \mathrm{~s}$, de séries temporelles de la composante longitudinale de la vitesse, nous avons estimé la densité spectrale d'énergie, après rééchantillonnage, par une méthode de Welch avec recouvrement, ce qui conduit à une résolution fréquentielle de $0,244 \mathrm{~Hz}[7,13]$. L'évolution du spectre d'énergie en fonction de la vitesse débitante $U_{\infty}$ (Fig. 9), révèle l'existence de plusieurs modes au sein de l'écoulement $[7,9]$. Pour $U_{\infty}<$ $1,4 \mathrm{~m} . \mathrm{s}^{-1}$, les résultats montrent l'existence de trois modes principaux (modes $0 ; 1 ; 2$ ). Pour $U_{\infty}>1,4 \mathrm{~m} . \mathrm{s}^{-1}$, les modes 0 et 1 disparaissent et le mode 2 persiste jusqu'à $U_{\infty}=2,3 \mathrm{~m} . \mathrm{s}^{-1}$ et on constate l'apparition du mode 3 . Ce dernier s'amplifie rapidement au-delà d'une vitesse de $2 \mathrm{~m} . \mathrm{s}^{-1}$ et persiste dans le domaine turbulent [7]. On remarque, également, que l'harmonique du mode 2 évolue, selon la vitesse $U_{\infty}$, entre 20 et $40 \mathrm{~Hz}$. La comparaison avec la formule de Rossiter [14], établie pour des vitesses bien supérieures, montre également un bon accord avec les fréquences mesurées. 


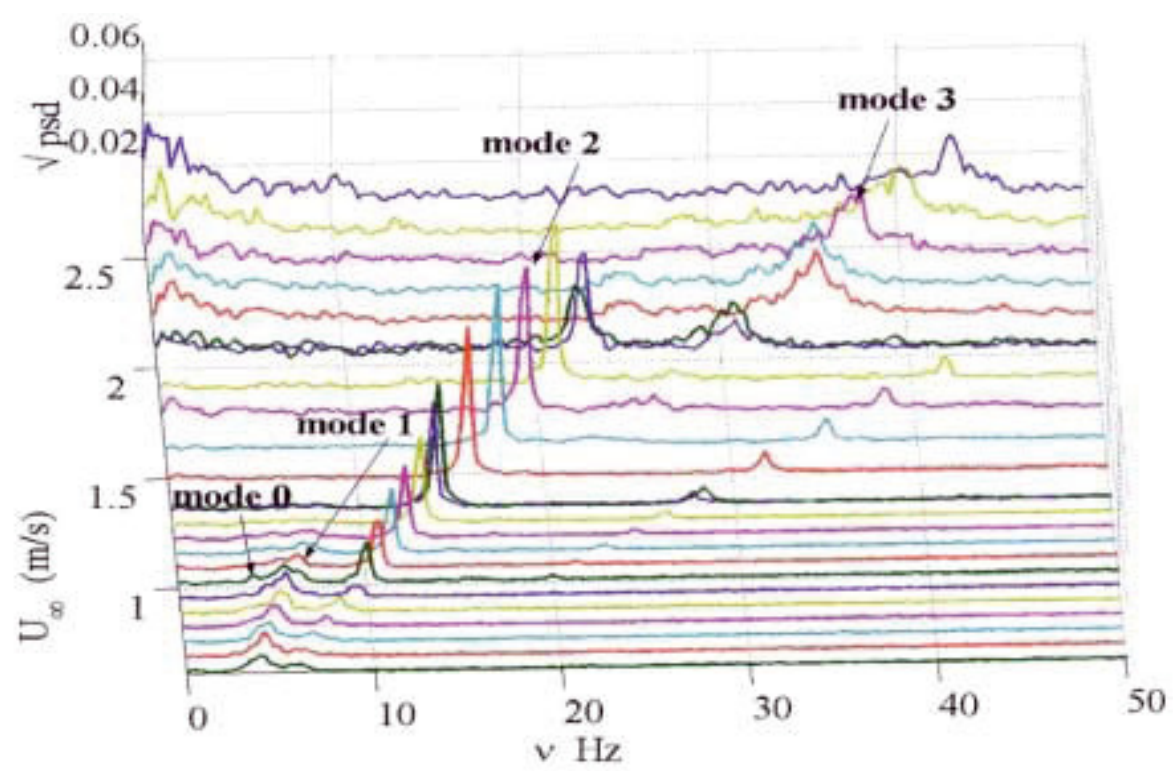

Fig. 9. Densité spectrale d'énergie de la composante horizontale de la vitesse, mesurée par LDV, en fonction de la vitesse $U_{\infty}$.

\subsection{Influence du rapport d'aspect sur les modes d'instabilités}

L'étude a été élargie à différents rapports d'aspect, en faisant varier seulement la longueur $L$ de la cavité. Quatre rapports d'aspect $[0,5 ; 1 ; 1,5 ; 2]$ ont été étudiés pour une vitesse débitante $U_{\infty}$ comprise entre $0,2 \mathrm{~m} . \mathrm{s}^{-1}$ et $5 \mathrm{~m} . \mathrm{s}^{-1}$.

La représentation des modes fréquentiels en fonction de la vitesse $U_{\infty}$ (Fig. 10), ne permet pas d'identifier les 3 modes déjà caractérisés pour $R=2$. En revanche on retrouve, pour chaque mode, l'existence d'un seuil de vitesse $U_{C}$ d'apparition des fréquences, ainsi qu'une évolution linéaire de la fréquence, quand on s'éloigne du seuil : $f=\alpha U_{\infty}+f_{0}$ ( $\alpha$ et $f_{0}$ sont identifiés par régression linéaire). Sur le tableau 4, nous donnons l'ensemble des résultats obtenus pour les quatre rapports d'aspect. Cela nous a conduits à représenter cette évolution, en fonction du nombre de Strouhal et du nombre de Reynolds.

\subsection{Détermination de la relation Strouhal Reynolds}

\subsubsection{Mise en équation}

On définit le nombre de Strouhal pour caractériser les modes dans la cavité $: S t=\frac{f L}{U_{\infty}}$. À partir de la relation $f=\alpha U_{\infty}+f_{0}$, le nombre de Strouhal s'écrit alors :

$$
S t=\frac{f L}{U_{\infty}}=\frac{\left(\alpha U_{\infty}+f_{0}\right) L}{U_{\infty}}=\frac{f_{0} L^{2}}{\nu} \frac{1}{R e_{L}}+\alpha L
$$

soit $S t=S t_{\infty}+\frac{\beta}{R e_{L}}$.

Le nombre de Strouhal est inversement proportionnel au nombre de Reynolds et atteint une valeur limite $S t_{\infty}$ pour les grandes valeurs du nombre de Reynolds. Pour chaque mode, on peut définir la valeur asymptotique remarquable $S t_{\infty}=\alpha L$ où $\alpha$ correspond à la pente de la

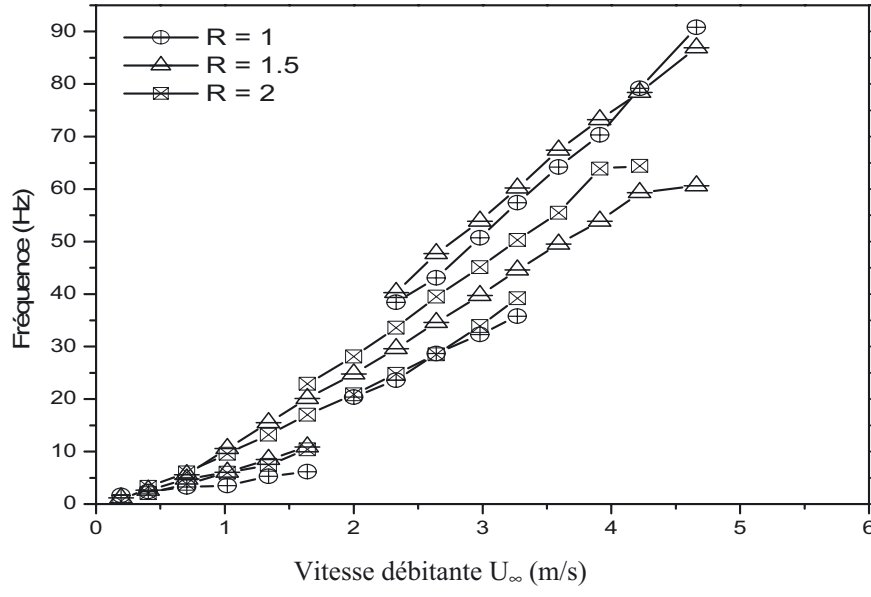

Fig. 10. Modes fréquentiels en fonction de $U_{\infty}$ pour les trois premières valeurs de $R$.

droite d'interpolation de la fréquence en fonction de la vitesse débitante. Notons ici que la loi donnant le nombre de Strouhal en fonction du nombre de Reynolds coïncide avec les solutions du modèle de Landau à coefficients complexes pour la bifurcation de Hopf $[11,15]$.

\subsubsection{Résultats expérimentaux}

Pour le calcul du nombre de Strouhal, nous avons tenu compte uniquement des modes associés aux séries de fréquences dont l'ajustement linéaire présente une ordonnée à l'origine négative. Nous pensons que les fréquences enregistrées associées à $f_{0}$ (positive) semblent représenter un autre phénomène oscillatoire qui pourrait être étudié ultérieurement. Le nombre de Strouhal, ainsi calculé est une fonction croissante du nombre de Reynolds 
Tableau 4. Résultats de l'ajustement linéaire $f=\alpha U_{\infty}+f_{0}$ pour les quatre cavités.

\begin{tabular}{|c|c|c|c|c|c|}
\hline & Modes & $\alpha \quad\left(\mathrm{m}^{-1}\right)$ & $f_{0}(\mathrm{~Hz})$ & $U_{C}=-\frac{f_{0}}{\alpha}\left(\mathrm{m} \cdot \mathrm{s}^{-1}\right)$ & $R e_{C}=\frac{U_{C} h}{\nu}$ \\
\hline \multirow{4}{*}{$R=0,5$} & mode 0 & 1,6589 & 1,7507 & - & - \\
\hline & mode 1 & 2,7451 & $-4,0306$ & 1,4680 & 9788 \\
\hline & mode 2 & 6,5513 & 4,2649 & - & - \\
\hline & mode 3 & $8 \times 10^{-4}$ & $14 \times 10^{-4}$ & - & - \\
\hline \multirow{4}{*}{$R=1$} & mode 0 & 3,0430 & 1,0350 & - & - \\
\hline & mode 1 & 6,6620 & 4,2290 & - & - \\
\hline & mode 2 & 12,4360 & $-4,7182$ & 0,3790 & 2526 \\
\hline & mode 3 & 22,4780 & $-15,7850$ & 0,7020 & 4680 \\
\hline \multirow{4}{*}{$R=1,5$} & mode 0 & 6,4900 & $-0,0634$ & 0,0097 & 64,6 \\
\hline & mode 1 & 7,4000 & 1,9770 & - & - \\
\hline & mode 2 & 14,6200 & $-4,0890$ & 0,2790 & 1860 \\
\hline & mode 3 & 19,9200 & $-5,2100$ & 0,2610 & 1740 \\
\hline \multirow{4}{*}{$R=2$} & mode 0 & 6,5600 & $-0,7500$ & 0,1140 & 762 \\
\hline & mode 1 & 7,4100 & 1,9100 & - & - \\
\hline & mode 2 & 12,1300 & $-2,5300$ & 0,2080 & 1391 \\
\hline & mode 3 & 17,0700 & $-5,6100$ & 0,3280 & 2192 \\
\hline
\end{tabular}

Tableau 5. Valeurs de $S t_{\infty}$ obtenues pour différentes cavités.

\begin{tabular}{ccccc}
\hline & $S t_{\infty}(R=0,5)$ & $S t_{\infty}(R=1)$ & $S t_{\infty}(R=1,5)$ & $S t_{\infty}(R=2)$ \\
\hline mode 1 & 0,068 & - & 0,480 & 0,656 \\
mode 2 & - & 0,621 & 1,096 & 1,213 \\
mode 3 & - & 1,123 & 1,490 & 1,707 \\
\hline
\end{tabular}

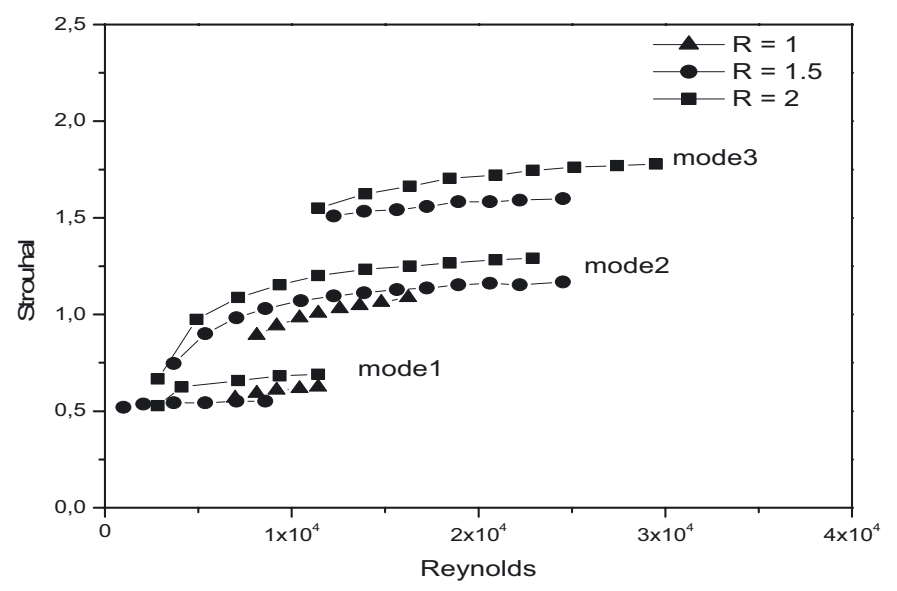

Fig. 11. Nombre de Strouhal en fonction du nombre de Reynolds : effet de regroupement en trois modes.

et tend vers une asymptote horizontale caractérisée par la valeur de $S t_{\infty}=\alpha L$.

Les différentes valeurs de $S t_{\infty}$ obtenues pour les différentes cavités et pour les différents modes observés, sont regroupées dans le tableau 5. Sur la figure 11, nous présentons la courbe de variation du nombre de Strouhal en fonction du nombre de Reynolds, avec effet de regroupement en 3 modes pour les différents rapports d'aspect.
Seul le mode 1 , pour la cavité $R=0,5$, présentant des valeurs très faibles, n'a pas été représenté. On constate une clarté dans le regroupement des différents modes y compris le mode 2 qui a été identifié, par les études antérieures, comme correspondant aux instabilités de couche de cisaillement [2]. Nous remarquons, pour un nombre de Reynolds supérieur à $10^{4}$, que l'analyse de ces résultats conduit bien à un regroupement pour chaque mode, permettant de bien séparer les modes les uns des autres [9]. Nous constatons également que pour une même cavité, le nombre de Strouhal augmente en moyenne de 0,5 lors du passage d'un mode au mode suivant.

\section{Conclusion}

Le travail effectué a pour but de caractériser expérimentalement les structures tourbillonnaires, prenant naissance dans l'interaction entre une couche limite et une cavité. La visualisation de l'écoulement dans la cavité a permis de mettre en évidence les différentes structures de l'écoulement. Afin de mieux quantifier les échappements tourbillonnaires observés, nous avons effectué en aval de la cavité des mesures par LDV de la composante longitudinale de la vitesse. Ces mesures ont été faites pour une large gamme de valeurs de la vitesse débitante, allant jusqu'à $5 \mathrm{~m} . \mathrm{s}^{-1}$, et pour plusieurs 
cavités caractérisées par différents rapports d'aspect. L'analyse de l'évolution du spectre d'énergie en fonction de la vitesse débitante, pour les différentes cavités étudiées, révèle l'existence de plusieurs modes, au sein de l'écoulement caractérisant chaque cavité. L'étude de la variation linéaire des fréquences en fonction des vitesses débitantes, nous a permis de définir une vitesse critique caractéristique de chaque mode fréquentiel. L'étude de l'évolution du système par la variation du nombre de Strouhal en fonction du nombre de Reynolds, nous a conduits à un regroupement pour chaque mode, permettant de bien séparer les modes les uns des autres. L'étude, par l'analyse modale, d'une loi de sélection de la longueur d'onde en fonction de la longueur de la cavité, est retenue comme une des perspectives de ce travail. Dans la continuité de cette étude, il serait aussi intéressant de faire des mesures du champ 3D, par la technique de vélocimétrie par images de particules pour différentes vitesses débitantes et pour différents rapports d'aspect de la cavité.

\section{Références}

[1] D. Rockwell, E. Naudascher, Self-sustained oscillations of impinging free shear layer, Ann. Rev. Fluid Mechanics 11 (1979) 67-94

[2] D. Rockwell, E. Naudascher, Self-sustaining oscillations of flow past cavities, J. Fluids Eng. 100 (1978) 152-165

[3] V. Sarohia, Experimental investigation of oscillations in flows over shallow cavities, AIAA J. 15 (1977) 984-991

[4] S. Ethembabaoglu, On the fluctuating flow characteristics in the vicinity of gate slots. Division of Hydraulic Engineering, University of Trondheim, Norvegian Institute of Technology, June 1973

[5] P. Huerre, M. Rossi, Hydrodynamics instabilities in open flows, Cambridge university Press 1998, Chap. 2, pp. 81294
[6] A. El Cafsi, Caractérisation expérimentale et étude fréquentielle des détachements tourbillonnaires issus de l'interaction couche limite-cavité, Thèse de doctorat d'état, université El Manar Tunisie, 2003

[7] F. Lusseyran, P. Gougat, A. Rambert, Y. Fraigneau, A. El Cafsi, Modes spatiaux et modes fréquentiels dans une cavité en interaction avec une couche limite : confrontation calcul-expérience à nombre de Reynolds modéré, $8^{\mathrm{e}}$ Congrès Francophone de vélocimétrie laser, Orsay, 2002, pp. 249-255

[8] A. El Cafsi, A. Rambert, P. Gougat, Optical flow technique used to characterise the flow inside different cavities interacting with a boundary layer. Euromech 406 Image Processing Methods in Applied Mechanics, Warsaw, Poland, 1999, pp. 83-86

[9] F. Lusseyran, P. Gougat, Y. Fraigneau, A. El Cafsi, Caractéristiques spatiales et temporelles de modes instables en cavité ouverte, $16^{\mathrm{e}}$ Congrès Français de mécanique, Nice, France, Cd cfm 2003

[10] L'anémométrie thermique et l'anémométrie Doppler à laser, Document technique, DISA, Denmark, 1980

[11] M.G. Bouchet, Étude expérimentale et numérique des auto-oscillations d'un jet confiné, Thèse de doctorat, Université Paris VI, 1996

[12] A. Maurel, P. Ern, B.J.A. Zielinska, J.E. Wesfreid, Experimental study of self-sustained oscillations in a confined jet, Phys. Rev. E. 54 (1996) 3643-3651

[13] A. Rambert, F. Lusseyran, P. Gougat, G. Quénot, Mesure 3D de vitesse par PIV flot optique basée sur un étalonnage simplifié des caméras, $8^{\mathrm{e}}$ Congrès Francophone de vélocimétrie laser, Orsay, 2002, pp. 403406

[14] J.E. Rossiter, Wind-Tunnel experiments on the flow over rectangular cavities at subsonic and transonic speeds, A.R.C. Reports and Memoranda, 3438, 1964

[15] M. Kiya, H. Ishikawa, H. Sakamoto, Near-wake instabilities and vortex structures of three-dimensional bluff bodies: a review, J. Wind Engineering and Industrial Aerodynamics 89 (2002) 1219-1232 